\title{
Stage IIIB Hilar Cholangiocarcinoma AJCC v8
}

National Cancer Institute

\section{Source}

National Cancer Institute. Stage IIIB Hilar Cholangiocarcinoma A/CC v8. NCI Thesaurus. Code C134749.

Stage IIIB includes: T4, N0, M0. T4: Tumor invading the main portal vein or its branches bilaterally, or the common hepatic artery; or unilateral second-order biliary radicals with contralateral portal vein or hepatic artery involvement. NO: No regional lymph node metastasis. M0: No distant metastasis. (AJCC 8th ed.) 